\title{
Effect of Acupressure on Thirst in Hemodialysis Patients
}

\author{
Li-Yu Yang ${ }^{a, b}$ Patsy Yates $^{d}$ Chi-Chun Chin ${ }^{a}$ Tsung-Kuei Kao ${ }^{c}$ \\ ${ }^{\text {a School of Nursing, }{ }^{\mathrm{b}} \text { College of Nursing, Kaohsiung Medical University, Kaohsiung, ' } D e p a r t m e n t ~ o f ~ N u r s i n g, ~}$ \\ Tajen University, Pingtung, Taiwan, ROC; ${ }^{\mathrm{d}}$ School of Nursing and Midwifery, Queensland University of Technology, \\ Brisbane, Qld., Australia
}

\section{Key Words}

Thirst $\cdot$ Salivary flow rate $\cdot$ Acupressure $\cdot$ Hemodialysis

\begin{abstract}
Background: Thirst and dry mouth are common among hemodialysis (HD) patients. This paper reports a study to evaluate the impact of an acupressure program on HD patients' thirst and salivary flow rates. Methods: The acupressure program included placebo, followed by true acupressure each applied for 4 weeks. Twenty-eight patients (mean age 57.6, $S D=16.13$ years) first received a sticker as placebo acupressure at two acupoints CV23 and TE17 three times a week for 4 weeks, and then received true acupressure in the same area for the next 4 weeks. Salivary flow rate and thirst intensity were measured at baseline, during and after treatment completion for both the placebo and true acupressure program. Results: The true acupressure program was associated with significantly increased salivary flow rate $(0.09 \pm 0.08$ $\mathrm{ml} / \mathrm{min}$ at baseline to $0.12 \pm 0.08 \mathrm{ml} / \mathrm{min}$ after treatments completion, $\mathrm{p}=0.04)$. The mean thirst intensity also improved from $4.21 \pm 2.66$ at baseline to $2.43 \pm 2.32(p=$ 0.008 ) after treatment completion in HD patients. There was no statistically significant difference in pre-post program salivary flow rate; however, significant improvement in thirst intensity scores was observed $(p=0.009)$ in the placebo acupressure program. Conclusion: This study provides preliminary evidence that acupressure may be effective in improving salivary flow rates and thirst intensity.
\end{abstract}

Copyright ๑ 2010 S. Karger AG, Basel

\section{KARGER}

Fax +4161306 1234 E-Mail karger@karger.ch www.karger.com

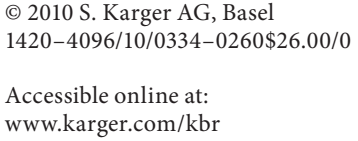

\section{Introduction}

Thirst or dry mouth is one of the most frequently occurring symptoms in hemodialysis (HD) patients. Studies report that $68.9-86 \%$ of HD patients experience exaggerated thirst and dry mouth [1-3]. The symptom of thirst can cause significant distress. Importantly, thirst is also associated with a higher large interdialytic weight gain (IWG) [3-6]. A large IWG can result in a much greater risk for cardiovascular morbidity, the main cause of death in end-stage renal disease (ESRD) patients [7].

Patients undergoing HD must follow specific treatment regimens, one of which is limiting fluid intake to avoid high IWG [8]. While adhering to fluid restriction is very important for HD patients, studies on the self-care behaviors involving HD patients from both western and eastern countries have indicated that between 32.6 and $74.6 \%$ of patients only adhere to recommended fluid intake $[4,9-12]$. There are likely to be a number of reasons why patients do not adhere to fluid restrictions, including the distress the patient may experience from severe thirst.

A limited number of studies have explored interventions to mange the symptom of thirst. Studies report that ESRD patients undergoing HD exhibit lower salivary flow rates than those of healthy subjects $[13,14]$. Acupuncture has been developed and tested to treat xerostomia in a number of studies [15-17]. One of these studies has reported that those patients with xerostomia who received the real acupuncture treatment showed a statisti- 
cally significant increased resting and stimulated salivary flow rate during and after the acupuncture treatment when compared with baseline [15]. Another study used an untreated control group and found that acupuncture only resulted in a significant increase in paraffinstimulated saliva secretion for patients with primary Sjögren's syndrome [17]. In other studies, the effectiveness of acupuncture with radiation-induced xerostomia has been shown to increase salivary flow rates and to reduce xerostomia intensity $[16,18,19]$. These findings suggest that acupuncture may be a useful treatment for the stimulation of salivary flow in xerostomia patients.

Unfortunately, acupuncture is an invasive procedure that must be delivered by licensed practitioners. Acupressure on the other hand, is a massage technique of Chinese origin that stimulates acupoints of the human body. Acupressure differs from acupuncture in that it is a therapy that puts pressure on acupoints on the surface of the body to relieve obstructions and to balance the energy flows; however, its effects are thought to be similar to those achieved by acupuncture. It is hypothesized that acupuncture can result in restoration of normal bodily function [20-22].

Acupressure uses hands as a tool to press acupoints on the skin. Patients can experience soreness, numbness, heaviness and fullness resulting from acupoint pressure. This subjective sensation can be experienced differently by individuals, and is referred to as 'De Qi' [21, 22]. Pressure is usually applied for a minimum of $15 \mathrm{~s}$, but it can last for between $30 \mathrm{~s}$ and $5 \mathrm{~min}$ [23]. The amount of pressure applied depends on patients' tolerance. Pressure can be applied until the patient experiences numbness, pressure, heaviness, soreness or a feeling of distention [21]. The effectiveness of acupressure in managing the symptom of thirst and improving salivary flow for patients has, however, not been tested. The present study was a single-blind and repeated-measures study to develop and undertake a preliminary evaluation of an acupressure strategy to stimulate salivary flow rates and improve HD patients' thirst symptoms.

\section{Patients and Methods}

Patients and Study Design

Patients were recruited from an HD center of a medical hospital in South Taiwan. All patients had had thirst symptoms in the last month and were undergoing regular HD three times a week. Patients with chronic heart failure and current skin problems (e.g. ulcer, rash) in the acupressure areas, or who were taking medications including tricyclic antidepressants, anticholinergics, an- tihistamines (with anticholingergic effect), and beta-blockers which may induce dry mouth, were excluded from the study.

An acupressure protocol was developed based on a review of the literature $[21,24,25]$ and in consultation with 5 licensed traditional Chinese physicians, who had graduated from medical schools in Taiwan or China and practiced Chinese medicine for more than 10 years. Two acupoints were used to increase salivary flow rate: Lianquan (CV23) on the anterior midline of the neck and Yifeng (TE17) posterior to both ear lobes. No previous study has documented the effects of acupressure on salivary flow rate in HD patients with thirst symptom. In this preliminary study, we therefore chose to evaluate whether acupressure could both increase salivary flow and decrease thirst intensity over time, by comparing a period of placebo intervention ('stickers' placed on the acupressure points) with a period of true acupressure (pressure delivered by the hands). The study design thus involved a single group with repeated measures involving a period of placebo intervention, followed by true acupressure, each applied for four weeks.

To standardize the intervention, principles of acupressure performance were defined. According to the literature, the power of pressure depends on patients' tolerance. Pressure is applied until the patient has numbness, pressure, heaviness, soreness or feeling of distention $[21,22]$. To determine the pressure to be applied in this study, 5 subjects not in the main study undergoing HD were chosen for a pilot study. Patients reported experiencing a 'De Qi' sensation when the pressure was maintained at $1-2 \mathrm{~kg}$. Therefore, $1-2 \mathrm{~kg}$ of finger pressure was applied in this study.

In order to enhance intra-rater reliability and to maintain the consistency of the pressure, prior to implementing the study, the finger pressure of 2 investigators was measured using a scale that had a unit of $20 \mathrm{~g}$ and maximum capacity of $6 \mathrm{~kg}$. The investigators were self-trained and the force of finger pressure was measured for each investigator over a 1-month period. The force of finger pressure was measured before the first subject was treated and after the last one was treated on the days of dialysis. The mean forces for all measurements were $1.96 \mathrm{~kg}(\mathrm{SD}=0.42)$ at first measure and 1.54 $\mathrm{kg}(\mathrm{SD}=0.28)$ at the end measure in true acupressure period.

Furthermore, for each investigator, 10 subjects not enrolled in the main study were selected to determine accuracy in selection of acupoints. The accuracy of the selection of two acupoints was determined by 2 experts who confirmed 100\% accuracy for each investigator.

For the first 4 weeks, all participants received 'a sticker' as placebo acupressure three times a week, and then received true acupressure three times a week to the same area for the last 4 weeks. Each acupoint lasted for $3 \mathrm{~min}$; a total of $9 \mathrm{~min}$ for each time. Salivary flow rate and thirst intensity were measured at baseline, during the intervention (after 3, 6, 9 treatments completion) and after completion of the 12 treatments in each of the placebo and true acupressure program.

The study was approved by the university and hospital human experimental and ethics committee. The researcher explained the study to potential participants, and written informed consent was obtained. Participants could withdraw at any time without adverse consequences.

Assessment of Salivary Flow Rates and Thirst Intensity Parameters

Demographic and medical data were obtained from each patient once informed consent had been obtained. The following 
Table 1. Patient characteristics at baseline $(n=28)$

\begin{tabular}{lc}
\hline Females/males & $14 / 14$ \\
Age, years & $57.61 \pm 16.13^{1}$ \\
Time on dialysis, months & $34.79 \pm 46.51^{1}$ \\
Thirst in the past month, scores & $5.54 \pm 3.13^{1}$ \\
IWG, kg & $2.45 \pm 1.14^{1}$ \\
Residual urine output & \\
$\quad$ Yes & \\
$<100 \mathrm{ml} /$ day & $12(42.9 \%)$ \\
$100-500 \mathrm{ml} /$ day & $8(28.6 \%)$ \\
$500-1,000 \mathrm{ml} /$ day & $2(7.1 \%)$ \\
No & $6(21.4 \%)$ \\
Educational level & $7(25 \%)$ \\
$\quad$ Primary school or lower & $4(14.3 \%)$ \\
Secondary school & $17(60.7 \%)$ \\
High school or higher & $9(32.1 \%)$ \\
Diabetes & $19(67.9 \%)$ \\
Yes & $18(64.3 \%)$ \\
No & $10(35.7 \%)$ \\
Chinese medicine experience & \\
Yes & $2(7.1 \%)$ \\
No & $26(92.9 \%)$ \\
Acupressure experience & \\
Yes & \\
No &
\end{tabular}

${ }^{1}$ Mean \pm SEM.

baseline characteristics were recorded: age, gender, religion, marital status, educational level, career, living condition, previous experience with Chinese medicine and acupressure, and medicine history, including period of HD, IWG, diabetes mellitus history, residual urine output (daily urine flow), thirst in the past month, salivary flow rate and thirst intensity.

The salivary flow rates were measured for unstimulated whole saliva, which was collected by oral cotton. The collection of whole saliva was performed under resting conditions in a hemoroom, at least $1 \mathrm{~h}$ after eating, smoking and drinking. Each saliva sample was collected over $10 \mathrm{~min}$ and measured by weight in grams. All samples were measured to an accuracy of $0.0001 \mathrm{~g}$ by the electric scale (Mettler B204S, Switzerland) presuming that $1 \mathrm{~g}$ of saliva is equivalent to $1 \mathrm{ml}$. Another instrument, a 10-point verbal rating scale ranging from 0 (no thirst) to 10 (worst thirst possible), was used to measure participants' intensity of thirst before, during and after treatment completion.

During the intervention period, all assessments were performed at pre-treatment (1 data point) and 1, 2, 3 weeks during the treatment period ( 3 data points) and after treatment completion (1 data point) on each placebo and true acupressure program. At each time point, salivary flow rate and thirst intensity for each participant was measured.

Statistical Analysis

Data were coded and analyzed using the SPSS version 16.0 statistical software package. Descriptive statistics were generated related to participant's characteristics and variables. A paired-sam- ples t test was conducted to compare baseline in salivary flow rate and thirst intensity measures before the placebo and true intervention program. To compare the changes for each outcome measure between baseline and 1 week, 2 weeks, 3 weeks after test and after treatment completion, generalized estimation equation (GEE) procedures were used. A level of $\mathrm{p}<0.05$ was considered statistically significant.

The parameters of the above statistical model were estimated with GEE to consider within-person variability. The GEE approach has been proposed as a non-parametric and appropriate method to conduct the repeated measurement analysis and to account for the correlated structure of disability data across repeated measurements. The main advantage of using GEE (when compared to maximum likelihood approaches) is that GEE is suitable for the analysis of both continuous and discrete outcome variables. Another advantage is that GEE seems to be quite robust against the working correlation structure, which must be assumed as correct for the within-subject correlations. In designs where $m=2$, the calculated odds ratios or coefficients with the GEE approach are exactly the same as they were calculated with multiple logistic or linear regression, but all available longitudinal data were used to calculate the risk in this model.

\section{Results}

A total of 28 subjects (14 male, 14 female) fulfilled the above-mentioned criteria and were enrolled in this program. All of the subjects lived with their families. Most subjects were married (95.2\%) and had religious beliefs (81\%). Most of the subjects were unemployed (82.1\%), 2 patients $(7.1 \%)$ were retired, and only 3 patients $(10.7 \%)$ were employed. A detailed description of the patients' baseline parameters in terms of mean age, sex, duration of dialysis treatment, thirst intensity in the past month, residual urine output, educational level, diabetes, IWG at baseline, previous experience with Chinese medicine and acupressure are presented in table 1.

Initial salivary flow rates were low and did not significantly change between baseline placebo and the true acupressure period measures $(\mathrm{t}=1.38, \mathrm{p}=0.18)$. The mean salivary flow rate at baseline was $0.10 \mathrm{ml} / \mathrm{min}$ $(\mathrm{SD}=0.09)$ with a range from $0.001-0.33 \mathrm{ml} / \mathrm{min}$. At baseline, half of the subjects $(50 \%)$ had a salivary flow rate lower than $0.10 \mathrm{ml} / \mathrm{min}, 12$ patients $(42.8 \%)$ had a range from 0.10 to $0.30 \mathrm{ml} / \mathrm{min}$, and only 2 patients $(7.2 \%)$ had a salivary flow rate above $0.30 \mathrm{ml} / \mathrm{min}$.

Prior to commencing the acupressure treatment program, a 10-point verbal rating scale was used to measure participants' intensity of thirst. At baseline, participant's reported a widely varying range of thirst intensity, with the majority of subjects having a moderate intensity of thirst. A total of 9 (32.2\%) patients had thirst intensity 
Table 2. Main variables at baseline and during acupressure treatment

\begin{tabular}{|c|c|c|c|c|c|c|}
\hline Variable & Baseline & Week 1 & Week 2 & Week 3 & $\begin{array}{l}\text { Treatment } \\
\text { completion }\end{array}$ & $\mathrm{p}$ \\
\hline \multicolumn{7}{|c|}{ Salivary flow rate, $\mathrm{ml} / \mathrm{min}$} \\
\hline Placebo & $0.10 \pm 0.09$ & $0.08 \pm 0.07$ & $0.08 \pm 0.07$ & $0.09 \pm 0.09$ & $0.10 \pm 0.08$ & 0.37 \\
\hline True & $0.09 \pm 0.08$ & $0.08 \pm 0.10$ & $0.07 \pm 0.07$ & $0.08 \pm 0.13$ & $0.12 \pm 0.08$ & 0.04 \\
\hline \multicolumn{7}{|l|}{ Thirst } \\
\hline Placebo & $4.75 \pm 2.53$ & $4.00 \pm 3.00$ & $3.96 \pm 2.69$ & $3.60 \pm 2.84$ & $3.21 \pm 2.48$ & 0.009 \\
\hline True & $4.21 \pm 2.66$ & $3.52 \pm 2.76$ & $3.63 \pm 2.62$ & $2.67 \pm 2.18$ & $2.43 \pm 2.32$ & 0.008 \\
\hline
\end{tabular}

scores at baseline of $\leq 3$ points, 13 (46.4\%) with scores of $4-6$ points, and $6(21.4 \%)$ with scores of $\geq 7$ points. The mean intensity of thirst was $4.75(\mathrm{SD}=2.53)$ with a range from 1 to 10 . Sixteen of the subjects (57.1\%) reported that their intensity of thirst had not changed over time, half of the subjects (52.4\%) felt thirsty all the time. Most of the subjects (82.1\%) used drinking to reduce their feeling of thirst, 4 of the subjects (14.3\%) used other methods to reduce thirst symptoms, such as chewing gum, and only one person (3.6\%) ignored the feeling of thirst.

At baseline, there was a significant association between thirst intensity and salivary flow rate $(r=-0.46$, $\mathrm{p}=0.01)$. Baseline measures of thirst intensity did not differ between the placebo and true intervention period $(t=$ $1.40, \mathrm{p}=0.17$ ).

The effects of acupressure are shown in table 2. In the placebo acupressure period, there was no statistically significant difference in treatment scores over the five time points for salivary flow rate $(p=0.37)$. However, there was significant difference in thirst intensity scores $(p=0.009)$ using GEE analysis after covariate adjustments for sex, age and time on dialysis. For the placebo acupressure group, this finding indicates no significant improvement in salivary flow rates, but a significant improvement in intensity of thirst scores is observed.

At the end of true acupressure phase of the study, the mean salivary flow rate was higher and thirst intensity was lower when compared with the baseline for the true acupressure period. Using GEE analysis after covariate adjustments for sex, age and time on dialysis, statistically significant differences in scores for salivary flow rate and thirst intensity over the five time points were observed ( $p=0.04, p=0.008$, respectively). Moreover, for the true acupressure group, the number of patients with xerostomia (salivary flow rate $<0.1 \mathrm{ml} / \mathrm{min}$ ) decreased from 17 patients at baseline to 12 patients at the end of the true acupressure period. Similarly, for the true acupressure group, the number of patients with thirst intensity scores more than 4 points decreased from 17 patients at baseline to 7 patients after true acupressure treatment completion. These differences noted for the true acupressure program were not observed for the placebo acupressure program.

\section{Discussion}

Our study has confirmed previous findings of a widespread low salivary flow rate in ESRD patients. Only 2 patients reach the normal range salivary flow rate of 0.3 $\mathrm{ml} / \mathrm{min}$ at baseline, with the mean salivary flow rates before acupressure program being $0.10 \pm 0.09 \mathrm{ml} / \mathrm{min}$. These results indicate that subjects in this study had more hyposalivation than reported in previous studies [4, 2627]. This may be attributed to the differences in sample inclusion criteria, since all participants in this study had thirst as a symptom. A negative correlation between thirst and low salivary flow rates has been documented in some studies in ESRD patients [3, 4]. This correlation, which was confirmed in our study, may indicate that the decrease in salivary flow rate in ESRD patients is greater in HD patients with thirst symptoms than in the ESRD population. The salivary flow rate is consistent with definitions of xerostomia, where salivary flow rate is defined as $<0.1 \mathrm{ml} / \mathrm{min}$ [28]. However, participants in this study did not report using methods to increase saliva production to relieve their thirst symptom, due to their disliking of the resulting taste. This means that half of the study subjects may have problems with eating dry food, and need to sip liquids to aid in swallowing food. Such increased fluid intake would increase IWG in HD patients.

The results of this study provide some evidence that acupressure on the acupoints CV23 and TE17 increased 
the salivary flow rates in HD population and are similar to the results obtained in studies using actual acupuncture techniques in improving symptoms related to radiation-induced xerostomia. The choice of acupressure points for the study was selected by 5 physicians who were certified in traditional Chinese medicine. CV23 and TE17 are mainly in the regions of the submandibular and labial glands; these acupoints have also been used in the acupuncture treatment of xerostomia studies [15-17]. The treatment schedule was modeled on the aforementioned studies in treating xerostomia, with a protracted course involving three weekly sessions for a total of twelve acupressure treatment sessions.

The underlying mechanism that has been proposed for acupuncture is that it works by affecting the autonomic nerve system to release neuropeptides which increase saliva [29]. Acupressure is thought to provide relief if the stimulation is sufficiently strong to stimulate the median nerve. It was assumed that improvements in the placebo acupressure should not be as good as those in the true acupressure group because the placebo group used a sticker on the pressure point, although the acupoints were the same. Moreover, this study showed that the salivary flow rates did not increase progressively during the true acupressure period, the salivary flow rate only increased after acupressure treatment completion. These results provide some evidence to suggest that participants may require a minimum of at least twelve sessions (that is three sessions per week for 4 weeks) of acupressure to reach the 'threshold' where the effect is present. A target volume of increase in salivary flow rates of around $\geq 0.1-$ $0.3 \mathrm{ml} / \mathrm{min}$ may be necessary to avoid the sensation of dry mouth [28]. Moreover, the twelve sessions of true acupressure used in this study have significantly increased the salivary flow rate, and as such it is not surprising to note that the thirst intensity in HD patients was also affected. The results from this study thus suggest that acupressure might have an important role in managing patients with low salivary flow rate and thirst. Unfortunately, the long-term effect was not followed so it was not known how long the effect can be maintained.

Our findings suggest that an acupressure treatment on points CV23 and TE17 that lasts for $3 \mathrm{~min}$, respectively, 3 days per week, for a total of 4 weeks can increase salivary flow rates, and decrease thirst intensity in HD patients.

\section{Limitations}

The methodological limitations of this study lie in the small sample size and the failure to measure the longterm effects. Moreover, as with most studies of these ther- apies, therapists are not blinded and as such may be subject to some bias. Also, while a repeated measure design was used, the internal validity might be affected due to practice effects associated with repeated uses of the VAS over time. Moreover, as all subjects received the placebo first, any inference about the effect of time cannot be assessed.

It is also acknowledged that while each participant was told that we would provide two methods for stimulating acupoints, and as such were blind as to which intervention was the 'true' intervention, participants are likely to have noticed reduced or no pressure with the placebo' stickers' stimulation on acupoints. As such, while participants were not informed that 'stickers' were a placebo intervention, they may have concluded that the 'true' acupressure was the 'true' intervention. This may explain our observation that the intensity of thirst after twelve sessions of 'a sticker' acupressure in the placebo acupressure period declined, as well as the differences observed between true and placebo interventions, reflecting a psychological reaction in some HD patients. That is, although the objective measures of salivary flow rate did not increase in the placebo time period, more subjective measures of thirst did show some changes. The substantial difference in thirst scores between the end of the placebo period and the commencement of the true intervention suggests, however, that any psychological reaction may not have a long-term effect.

Although we concluded in this preliminary study that acupressure may be a valuable method for the stimulation of salivary secretion in many HD patients with thirst, further research is required using a crossover design, and a more effective placebo involving pressing sham acupoints not related to salivary flow rate or thirst as a control treatment instead of a sticker maneuver. In addition, almost all the patients felt discomfort and could not tolerate over $3 \mathrm{~min}$ of pressure on each acupoint. It might be preferable to use a different treatment to stimulate the acupoints, such as TENS to make patients more comfortable.

We recommend that the methodological limitations of this study and different treatments should be considered in future studies.

\section{Acknowledgments}

The authors thank Dr. Kuan-Chia Lin for his assistance in the study statistic. This work was supported by a research grant from the National Science Council of Taiwan (NSC-92-2314-B-037097). 


\section{References}

1 Giovannetti S, Barsotti G, Cupisti A, et al: Dipsogenic factors operating in chronic uremics on maintenance hemodialysis. Nephron 1994;66:413-420.

$\checkmark 2$ Kho HS, Lee SW, Chung SC, Kim YK: Oral manifestations and salivary flow rate, $\mathrm{pH}$, and buffer capacity in patients with endstage renal disease undergoing hemodialysis. Oral Surg Oral Med Oral Pathol Oral Radiol Endod 1999;88:316-319.

-3 Sung JM, Kuo SC, Guo HR, et al: Decreased salivary flow rate as a dipsogenic factor in hemodialysis patients: evidence from an observational study and a pilocarpine clinical trial. J Am Soc Nephrol 2005;16:3418-3429.

4 Bots CP, Brand HS, Veerman EC, et al: Interdialytic weight gain in patients on hemodialysis is associated with dry mouth and thirst. Kidney Int 2004;66:1662-1668.

$\checkmark 5$ Flanigan M: Dialysate composition and hemodialysis hypertension. Semin Dial 2004; 17:279-283

-6 Kugler C, Vlaminck H, Haverich A, Maes B: Nonadherence with diet and fluid restrictions among adults having hemodialysis. J Nurs Scholarsh 2005;37:25-29.

-7 Kalantar-Zadeh K, Regidor DL, Kovesdy CP, et al: Fluid retention is associated with cardiovascular mortality in patients undergoing long-term hemodialysis. Circulation 2009;119:671-679.

8 Mallick NP, Gokal R: Haemodialysis. Lancet 1999;353:737-742.

-9 Durose CL, Holdsworth M, Watson V, et al: Knowledge of dietary restrictions and the medical consequences of noncompliance by patients on hemodialysis are not predictive of dietary compliance. J Am Diet Assoc 2004;104:35-41.
10 Lee SH, Molassiotis A: Dietary and fluid compliance in Chinese hemodialysis patients. Int J Nurs Stud 2002;39:695-704.

11 Lee Y, Wang R: Helplessness, social support and self-care behaviors among long-term hemodialysis patients. J Nurs Res 2001;9:147158.

12 Pang SK, Ip WY, Chang AM: Psychosocial correlates of fluid compliance among Chinese haemodialysis patients. J Adv Nurs 2001;35:691-698.

13 Kao CH, Hsieh JF, Tsai SC, Ho YJ, Chang HR: Decreased salivary function in patients with end-stage renal disease requiring hemodialysis. Am J Kidney Dis 2000;36:1110 1114.

14 Kaya M, Cermik TF, Ustun F, Sen S, Berkarda S: Salivary function in patients with chronic renal failure undergoing hemodialysis. Ann Nucl Med 2002;16:117-120.

15 Blom M, Dawidson I, Angmar-Mansson B: The effect of acupuncture on salivary flow rates in patients with xerostomia. Oral Surg Oral Med Oral Pathol 1992;73:293-298.

16 Blom M, Dawidson I, Fernberg JO, Johnson G, Angmar-Mansson B: Acupuncture treatment of patients with radiation-induced xerostomia. Eur J Cancer B Oral Oncol 1996; 32B:182-190.

17 List T, Lundeberg T, Lundstrom I, Lindstrom F, Ravald N: The effect of acupuncture in the treatment of patients with primary Sjogren's syndrome. A controlled study. Acta Odontol Scand 1998;56:95-99.

18 Johnstone PA, Niemtzow RC, Riffenburgh RH: Acupuncture for xerostomia: clinical update. Cancer 2002;94:1151-1156.

19 Johnstone PA, Peng YP, May BC, et al: Acupuncture for pilocarpine-resistant xerostomia following radiotherapy for head and neck malignancies. Int J Radiat Oncol Biol Phys 2001;50:353-357.
20 Lin WJ, Wu MC: Advancements in research of channel and network vessels (in Chinese). J Chin Clin Tradit Med 2004;10:338-344.

$21 \mathrm{Ma} \mathrm{H}$, Chang M, Lin C: A systematic review of acupressure for the application on nursing practice (in Chinese). J Nurs 2007;54:35-44.

22 Maa S: Application of acupressure in nursing practice (in Chinese). J Nurs 2005;52:5-10.

23 Matsumura WM: Use of acupressure techniques and concepts for nonsurgical management of TMJ disorders. J Gen Orthod 1993;4:5-16.

24 Brennan MT, Shariff G, Lockhart PB, Fox PC: Treatment of xerostomia: a systematic review of therapeutic trials. Dent Clin North Am 2002;46:847-856.

25 Blom M, Lundeberg T: Long-term follow-up of patients treated with acupuncture for xerostomia and the influence of additional treatment. Oral Dis 2000;6:15-24.

26 Bots CP, Brand HS, Veerman EC, et al: The management of xerostomia in patients on haemodialysis: comparison of artificial saliva and chewing gum. Palliat Med 2005; 19: 202-207.

-27 Bayraktar G, Kazancioglu R, Bozfakioglu S, et al: Stimulated salivary flow rate in chronic hemodialysis patients. Nephron 2002;91: 210-214.

28 Dawes C: How much saliva is enough for avoidance of xerostomia? Caries Res 2004; 38:236-240.

29 Yao T: Acupuncture and somatic nerve stimulation: mechanism underlying effects on cardiovascular and renal activities. Scand J Rehabil Med Suppl 1993;29:7-18. 